\title{
Representaciones sociales de un grupo de estudiantes universitarios colombianos sobre el ambiente'
}

\author{
Alejandro Rico Méndez"
}

\section{Resumen}

En el ámbito social existe una relación bidireccional muy fuerte entre lo que los sujetos piensan, dicen y hacen. Precisamente por ello, teniendo en cuenta la importancia cada vez mayor de los temas ambientales en ámbito de la educación superior, en este trabajo se abordó dicho tópico desde la perspectiva de las representaciones sociales y la sociolingüística. Puntualmente, el objetivo de la presente investigación fue caracterizar las representaciones sociales de un grupo de estudiantes universitarios bogotanos sobre el ambiente. Esto se hizo desde un enfoque cualitativo, usando los referentes teóricos y metodológicos de las representaciones sociales y la sociolingüística. Para el análisis de las representaciones del ambiente se utilizó la caracterización propuesta por el profesor y experto en la materia Marcos Reigota. Desde tales referentes, la indagación concluye que en los discursos de estos estudiantes, prevalece una representación social antropocéntrica y sistémica del ambiente. La primera se evidencia cuando se observa que en la mayoría de los discursos analizados, la naturaleza suele importar solo como medio para el bienestar humano. La segunda, cuando se aprecia que en casi todos estos discursos, la problemática ambiental aparece estrechamente ligada a las dinámicas ecológicas, socioculturales y político-económicas. El artículo finaliza proponiendo que la categoría representación sistémica del ambiente, puede subdividirse en dos categorías, Sencillas y Complejas, según el nivel de profundidad alcanzando en el análisis del tema ambiental por cada una de ellas; y que es fundamental tener en cuenta el contenido de dichas representaciones al diseñar propuestas de educación ambiental.

\section{Palabras Clave}

I- Agradecimientos al profesor Luis Eduardo Florez, Coordinador de la Especialización en Docencia Universitaria de la Universidad Cooperativa de Colombia, por su apoyo y paciencia incondicional en el desarrollo de esta investigación.

II- Universidad Nacional de Colombia, Bogotá, Colombia.

Contacto: alexrico_50@yahoo.com
Representaciones sociales - Sociolingüística - Educación ambiental. 


\title{
Social representations of a group of Colombian university students about the environment'
}

\author{
Alejandro Rico Méndez"
}

\begin{abstract}
In the social field, there is a strong bidirectional relation between what individuals think, say, and do. Precisely for this reason, considering the growing importance of environmental issues in higher education, this study addresses them from the perspective of social representations and sociolinguistics. Its specific objective was to characterize the social representations that a group of university students in Bogotá city has about the environment. This was done from a qualitative perspective, using the theoretical and methodological framework of social representations and sociolinguistics. For the analysis of the representations of the environment, we used the characterization proposed by professor and expert in the field Marcos Reigota. The inquiry concluded that, in the discourses of these students, an anthropocentric and systemic social representation of the environment prevails. The first is evident when it is observed that, in most of the discourses analyzed, nature usually matters only as a means for human welfare. The second is clear when it is observed that, in most of these discourses, environmental issues appear closely tied to the environmental, sociocultural, and political-economic dynamics. The article concludes by proposing that the category Systemic Representation of the Environment can be subdivided into two sub-categories, Simple and Complex, depending on the depth reached in the analysis of environmental issues for each one. This research also indicates that it is essential to consider the content of these representations in the design of environmental education programs.
\end{abstract}

\section{Keywords}

Social representations - Sociolinguistics - Environmental

I- Agradecimientos al profesor Luis Eduardo education.

Florez, Coordinador de la Especialización en Docencia Universitaria de la Universidad Cooperativa de Colombia, por su apoyo y paciencia incondicional en el desarrollo de esta investigación.

II- Universidad Nacional de Colombia, Bogotá, Colombia.

Contacto: alexrico_50@yahoo.com 


\section{Introducción}

Dada la gravedad de la crisis ambiental contemporánea, cada vez más personas están de acuerdo con la importancia de la educación ambiental dentro de los procesos de formación integral que se adelantan en los diversos ámbitos educativos. Por ello desde la Cumbre Mundial sobre Desarrollo Sostenible celebrada en Rio durante el 1992, se les solicitó a todas las instituciones de educación, incluidas desde luego las de educación superior, adelantar acciones en beneficio del ambiente desde la docencia, la investigación y la extensión, tal como por ejemplo se puede apreciar en el capítulo 37 de la Agenda 21.

A partir de allí, se han venido adelantando diferentes acciones para avanzar en el cumplimiento de estos objetivos, entre ellas varios estudios desde el campo de las representaciones sociales, pues como lo señala Marcos Reigota, el "primer paso para la realización de la educación ambiental consiste en la identificación de las representaciones que tienen las personas involucradas en el proceso educativo" (1994, p. 14 apud FERREIRA, 2002, p.24). Esto debido a que es muy difícil avanzar en los procesos de cualificación académica, cuando se desconocen las representaciones sociales que los actores directamente involucrados en estas dinámicas tienen sobre la problemática ambiental.

Precisamente de aquí surge la necesidad de caracterizar una parte del universo simbólico que orienta las acciones de estos actores, para a partir de allí diseñar acciones educativas, que permitan mejorar el proceso de formación en esta área de trascendental importancia para el país. Concretamente, el objetivo de esta investigación fue caracterizar las representaciones sociales que los estudiantes inscritos en la Corporación Tecnológica de Bogotá (CTB) en el año 2013 tienen sobre el ambiente, pues se parte del presupuesto de que dichas concepciones influyen profundamente, en la forma en la que estos sujetos actúan frente a este tipo de situaciones.

\section{Marco teórico}

A continuación se explicarán algunos de los referentes conceptuales más importantes que fundamentaron teórica y metodológicamente el desarrollo de este estudio.

\section{Sistemas ambientales y educación ambiental}

En esta investigación, se entenderá el ambiente, como aquel sistema complejo producto de la interacción entre los sistemas naturales (físicos, químicos y biológicos) y los sistemas sociales (culturales, económicos, políticos y religiosos) (CARRISOZA, 2000). Los sistemas ambientales, son una definición más concreta para el caso ambiental del término sistema complejo, los cuales requieren por su misma naturaleza de investigaciones interdisciplinarias para su estudio, ya que son sólo estas las que permiten captar su estructura y funcionamiento complejo, pues las interrelaciones entre la totalidad y las partes, que es lo que define a estos sistemas, no pueden ser estudiadas mediante el fraccionamiento del sistema en subsistemas parciales (GARCÍA, 1994).

En el contexto colombiano se ha definido la educación ambiental "como el proceso que le permite al individuo comprender las relaciones de interdependencia con su entorno ambiental, a partir del conocimiento reflexivo y crítico de su realidad biofísica, social, política, económica y cultural" (BERMÚDEZ, 2003, p. 24). Desde este enfoque se puede afirmar que en Colombia, a la educación ambiental aun le queda un largo camino por recorrer sobre todo en el ámbito universitario (ÁNGEL, 2001). Esto se debe, entre otras cosas, a que hace falta una mayor capacitación y actualización de la comunidad educativa en diversas áreas del conocimiento comenzando por la ambiental, la pedagógica y la investigativa. Como ya se dijo, esta investigación busca contribuir a llenar parte de ese vacío. 


\section{¿Qué son las representaciones sociales?}

Enseguida se presentan algunos de los referentes más importantes de la teoría de las representaciones sociales con el propósito de ubicar teórica y conceptualmente al lector en la temática, y de vislumbrar sucintamente el enorme potencial que este enfoque posee para ayudar a comprender los fenómenos educativos. En todo caso, se aclara que no se pretende hacer una descripción exhaustiva de la compleja y rica gama de matices epistemológicos, teóricos y metodológicos que distinguen esta área de estudio. Las personas que deseen hacer esto, pueden remitirse directamente a las referencias bibliográficas de este trabajo, en donde podrán profundizar en las ideas aquí esbozadas.

Las representaciones sociales fueron definidas explícitamente por primera vez en la literatura académica por Serge Moscovici, quien las conceptualizó como:

Un corpus organizado de conocimientos y una de las actividades psíquicas gracias a las cuales los hombres hacen inteligible la realidad física y social, se integran en un grupo o en una relación cotidiana de intercambios, liberan los poderes de su imaginación... son sistemas de valores, nociones y prácticas que proporciona a los individuos los medios para orientarse en el contexto social y material, para dominarlo (MOSCOVICI, 1979 apud PERERA, 1999, p. 10).

A partir de esta definición, innumerables autores han venido dándole diversos matices al concepto, de acuerdo a su formación e intereses investigativos. Así por ejemplo Denise Jodelet, discípula de Moscovici y una de las autoras más reconocidos en este campo, las definió así:

Las representaciones sociales conciernen al conocimiento de sentido común que se pone a disposición en la experiencia cotidiana; son programas de percepción, construcciones con status de teoría ingenua, que sirven de guía para la acción e instrumento de lectura de la realidad; sistemas de significaciones que permiten interpretar el curso de los acontecimientos y las relaciones sociales; que expresan la relación que los individuos y los grupos mantienen con el mundo y los otros; que son forjadas en la interacción y el contacto con los discursos que circulan en el espacio público; que están inscritas en el lenguaje y en las prácticas; y que funcionan como un lenguaje en razón de su función simbólica y de los marcos que proporcionan para codificar y categorizar lo compone el universo de la vida. (JODELET, 2000 apud PERERA, 2005, p. 47).

De otro lado, Azevedo escribe lo siguiente:

[...] una representación social es una teoría contemporánea que trata de comprender cómo, tanto individuo como colectividad, interpretan los fenómenos sociales. Es fragmentada y parcial; tiene que ver con las visiones del mundo, con las ideologías, con el sentido común, con la divulgación delas ideas, con el conocimiento. Las representaciones sociales circulan, comunican cómo se ve una temática determinada y reflejan un concepto sociohistórico cultural en el cual se inscribe el sujeto (AZEVEDO, 1999, p. 70 apud 2002, p. 24).

Como se puede inferir de las definiciones anteriores, la teoría de las representaciones sociales, se encuentra a medio camino entre los fenómenos psicológicos y los socioculturales, lo que hace que en su fundamentación conceptual necesariamente intervengan elementos de la psicología, la sociología y la antropología (BANCHS, 2000). A través de ellas es posible estudiar los fenómenos sociales atendiendo simultáneamente sus factores subjetivos, intersubjetivos y objetivos, cuya interacción constituye la esencia de la realidad socialmente construida. 
En varias partes del mundo se ha utilizado la teoría de las representaciones sociales para estudiar las temáticas ambientales. En Latinoamérica, el profesor Marcos Reigota, consideró que dada la diversidad de concepciones existentes sobre el concepto de ambiente, era fundamental caracterizar las representaciones existentes a su alrededor, para a partir de allí diseñar propuestas de intervención educativas. Por ello, propuso clasificar las representaciones sociales sobre el ambiente de la siguiente forma:

- Representación naturalista: Se caracteriza por enfatizar los aspectos naturales, en ocasiones mezclando conceptos de la ecología.

- Representación globalizadora: Se caracteriza por las relaciones recíprocas entre ambiente natural y sociedad.

- Representación antropocéntrica: Se caracteriza por el empleo de los recursos naturales como garantía de la sobrevivencia humana (REIGOTA, 1994 apud MOLFI, 2000, p. 38) ${ }^{1}$.

A partir de este trabajo se han venido desarrollando diversas investigaciones siguiendo esta línea a lo largo de toda la región (AZEVEDO, 1999; OKAMURA, 1996; MOLFI, 2000 apud FERREIRA, 2002, p. 26).

\section{La sociolingüística y las representaciones sociales}

Las representaciones sociales se constituyen mediante los intercambios lingüísticos, sociales y culturales que tienen lugar en la vida cotidiana, en un proceso de interacción en donde las prácticas y las representaciones se influencian recíprocamente (CISRS, 2010). Debido al papel fundamental que tiene el lenguaje en su constitución (ALFONSO, 2011), en esta investigación se optó por utilizar el análisis discursivo para identificar y

1- Originalmente Reigota utiliza el término globalizantes para designar a estas representaciones, pero en este artículo se denominaran sistémicas para evitar confusiones con el concepto de globalización. caracterizar las representaciones sociales que los estudiantes tenían sobre el ambiente.

Para llevar a cabo esta labor, se empleó la propuesta de Pierre Bourdieu, sobre el análisis sociolingüístico de las prácticas discursivas. Para este autor, el discurso es un tipo específico de acción social, que como tal posee un sentido y sirve como medio para la satisfacción de ciertos fines (comunicar, persuadir, ordenar, etc.). Éste es protagonizado por actores sociales reales en contextos históricos concretos, cuya configuración específica da lugar a determinados campos, habitus y mercados lingüísticos, que son los elementos que en su conjunto, explican parcialmente el contenido, la estructura, y verdadera intencionalidad de los discursos (BOURDIEU, 1985 apud RICO, 2004).

La propuesta de Bourdieu básicamente busca dar cuenta de las relaciones existentes entre las condiciones sociales de producción y circulación de los discursos con sus propiedades internas, valiéndose para ello de la utilización de los conceptos de Habitus Lingüístico (que haría referencia básicamente al perfil institucional y génesis histórica de la organización que emite el discurso), Capital Lingüístico (reconocimiento y autoridad con que ésta cuenta para emitirlo, lo que depende de su lugar dentro del campo social), y Mercado Lingüístico (rasgos generales de las personas o instituciones a quienes se dirigen). En este marco "resulta imposible interpretar un acto de comunicación dentro de los límites de un análisis meramente lingüístico" (BOURDIEU; WACQUANT, 1995, p. 104), pues como lo ha demostrado la sociolingüística, lo esencial de la comunicación se encuentra en los contextos donde esta se desarrolla (BOURDIEU, 2000). De acuerdo con la sociolingüística, el lenguaje no sólo contribuye a entretejer pensamientos, sino que además tiene una función primordial en la construcción social de la realidad (CHRISTIDOU; DIMOPOULOS; KOULAIDIS, 2004).

Desde luego, la sociolingüística es totalmente a fin a la naturaleza de las representaciones sociales, pues de acuerdo con Ibette Alfonso Pérez: 
Cuando nos referimos al contexto sociocultural que determina una representación, debemos tener en cuenta las condiciones históricas, económicas e ideológicas en que surgen, se desarrollan $\mathrm{y}$ desenvuelven los grupos y objetos de representación que estudiamos. Son importantes además, las instituciones $\mathrm{u}$ organizaciones con las que interactúan los sujetos y grupos, así como la inserción social de los individuos en términos de pertenencia a determinados grupos $\mathrm{y}$ las prácticas sociales en los que estos participan (2011, p. 8).

\section{Metodología}

Por su naturaleza interdisciplinar, las representaciones sociales han sido estudiadas desde muy diversas perspectivas, mediante el uso de gran variedad de técnicas cualitativas y cuantitativas (CISRS, 2010). Concretamente, esta investigación se desarrolló desde una perspectiva no experimental, mediante la utilización de un método de investigación esencialmente cualitativo, por ser este uno de los caminos más apropiados para dar cuenta de la complejidad de los fenómenos educativos, especialmente de los referidos a las representaciones sociales (BANCHS, 2000). De forma puntual, se analizaron sociolingüísticamente los discursos que los participantes estructuraron en torno al ambiente, para a partir de allí, dar cuenta de sus respectivas representaciones sociales.

Los discursos se obtuvieron mediante la realización de paneles de discusión que se organizaron alrededor de la siguiente batería de preguntas, la cual fue cuidadosamente construida a partir de las reflexiones desarrolladas en el marco teórico. Luego de realizar el pilotaje de este instrumento, sólo se dejaron aquellos interrogantes que generaron más controversia y discusión entre los participantes:

1. ¿Qué es el ambiente?

2. ¿Crees que el desarrollo social y el cuidado ambiental son incompatibles?
3. Según tu opinión ¿Qué responsabilidad tienen las empresas, las instituciones educativas, el gobierno y la ciudadanía frente a la problemática ambiental?

4. ¿Existe algún tipo de relación entre tu campo de acción profesional y la problemática ambiental?

5. ¿Qué factores consideras más importantes a la hora de tratar de solucionar esta problemática? Los científicos-técnicos, los económicos, los culturales. Otro ¿cuál? ¿Por qué?

6. ¿En los próximos veinte años las situaciones ambientales van a mejorar o a empeorar?

7. ¿Puntualmente qué haces a favor del ambiente?

Este trabajo por cuestiones de extensión, se concentrará en las discusiones que tuvieron lugar alrededor de la primera pregunta, retomando eventualmente algunos elementos de las respuestas de los demás interrogantes para enriquecer su análisis.

La población objeto de estudio, estuvo constituida básicamente por los estudiantes matriculados en la CTB durante el segundo semestre del 2013. Ésta es una institución de educación superior, con más de 50 años de fundada y cuyos principales programas son Tecnología en Regencia de Farmacia y Tecnología en Química Industrial. Su misión es:

[...] la formación de profesionales integrales (en lo humano, ético, moral, científico, investigativo, tecnológico, cultural, empresarial, industrial, ambiental y relación con el entorno), fundamentada en una sólida formación investigativa, soportada en un modelo pedagógico caracterizado por la excelencia académica, la indagación y la búsqueda sistemática en las diferentes áreas del conocimiento en el ámbito local, regional, internacional (CTB, 2013).

Los alumnos de los diversos programas que ofrece la CTB, son en su mayoría jóvenes entre los 16 y los 22 años pertenecientes a 
los estratos dos y tres. La mayor parte de los matriculados en la jornada nocturna, trabaja $\mathrm{y}$ estudia al mismo tiempo, en actividades relacionadas con el sector de la salud y la química. De este grupo se seleccionó una muestra representativa, utilizando los siguientes parámetros cualitativos: a) Que estuvieran cursando materias de I o II semestre; b) Que mostraran cierto interés por las temáticas ambientales; y c) Que tuvieran facilidad para expresarse de forma verbal o escrita.

En total se realizaron seis paneles de discusión (tres con estudiantes de la jornada de la mañana y tres con estudiantes de la jornada de la noche; tres del programa de Regencia de Farmacia y tres de Química Industrial), cada uno con un promedio de quince estudiantes y una duración aproximada de 45 minutos. Se procuró que cada panel estuviera constituido equitativamente por hombres y mujeres. Para su realización, se reunió al grupo en un salón y después de una breve explicación respecto a la naturaleza del estudio, se leyeron una por una las preguntas del cuestionario, dándoles el tiempo para que expresaran libremente su punto de vista.

Siendo consecuente con los referentes teórico-metodológicos propuestos con anterioridad, en el análisis de los discursos recopilados, se procuró tener en cuenta los contextos socioculturales, políticoeconómicos y por supuesto ambientales donde tales representaciones y discursos tuvieron lugar, ya que como se ha dicho, estas circunstancias condicionan su contenido y estructura. Para facilitar la caracterización sociolingüística de los discursos por parte de lector, cada vez que se transcriba uno de los fragmentos de los paneles de discusión, se ofrecerá la siguiente información sobre el emisor: género, edad, programa de estudio y jornada. Adicionalmente, cuando sea necesario se hará alusión a los aspectos socioculturales $\mathrm{y}$ ambientales que puedan estar afectando el discurso analizado.

\section{Análisis y discusión}

\section{Representaciones sociales antropocéntricas del ambiente}

Desde la tipología propuesta por el profesor Marcos Reigota sobre las representaciones sociales del ambiente (1994 apud MOLFI, 2000), se puede afirmar que una de las características que comparten sin excepción los discursos analizados, es el enfoque antropocéntrico desde el cual el hombre es el centro de todas las cosas y la naturaleza un simple recurso a su servicio. Es decir, en todos los discursos, en mayor o en menor grado, se aboga por conservar y preservar la naturaleza, por aprovecharla dentro de los parámetros del desarrollo sostenible, pero no porque ésta tenga un valor en sí misma, porque merezca respeto, porque posea derecho a existir por sí sola, sino porque el bienestar a largo plazo de la humanidad depende del uso racional que de ella se haga. En todos estos discursos, naturaleza es igual a recurso natural, tal como se puede observar enseguida:

Hombre/17/Farmacia/Diurno: el ambiente son todos los recursos naturales que nos rodean como el agua, el aire, la madera y cosas así y de los que nosotros dependemos para nuestra supervivencia, para nuestras necesidades.

A pesar de que se menciona explícitamente muy pocas veces, es evidente que la mayoría de estas representaciones sociales, se estructuran alrededor del desarrollo sostenible, el cual se entiende a grandes rasgos como el aprovechamiento racional de los recursos naturales que no compromete las necesidades de las generaciones futuras. Esto no significa sin embargo, que entre los panelistas exista consenso respecto a la forma de operacionalizar dicho concepto. Esto se puede apreciar aquí: 
Mujer/18/Química/Nocturna: Se debe fomentar en los estudiantes la conciencia ecológica, el cuidado el medio ambiente, que es lo que les va a quedar realmente a ellos y a la generación que sigue.

Este antropocentrismo, se explica parcialmenteporel hecho dequetodoslosestudiantes han sido educados en ámbitos socioculturales en donde a través de los diferentes procesos de socialización se estructura una representación social, que explícita o implícitamente, supone que los seres humanos son el centro de todas las cosas y que por ende el mundo y todos sus recursos están al servicio de la humanidad (RICO, 2009).

Esta representación antropocéntrica del ambiente fue analizada por el ex-profesor de la Universidad de California Lynn White Jr, en un artículo de la revista Science. Allí, este investigador identificó dos formas de ver el mundo de origen religioso, que debido a su interpretación favorecieron la conformación de dos de las causas estructurales más importantes de la actual crisis ambiental: el antropocentrismo propio de la cultura occidental y su consecuente ruptura con el orden natural. Con respecto al primer punto, en dicho trabajo, textualmente se puede leer lo siguiente:

By gradual stages a loving and allpowerful God had created light and darkness, the heavenly bodies, the earth and all its plants, animals, birds, and fishes. Finally, God had created Adam and, as an afterthought, Eve to keep man from being lonely. Man named all the animals, thus establishing his dominance over them. God planned all of this explicitly for man's benefit and rule: no item in the physical creation had any purpose save to serve man's purposes. And, although man's body is made of clay, he is not simply part of nature: he is made in God's image (WHITE, 1967, p. 1205).

En lo concerniente al segundo punto, escribe:
Especially in its Western form, Christianity is the most anthropocentric religion the world has seen [...]. Man shares, in great measure, God's transcendence of nature. Christianity, in absolute contrast to ancient paganism and Asia's religions (except, perhaps, Zorastrianism), not only established a dualism of man and nature but also insisted that it is God's will that man exploit nature for his proper ends (1967, p. 1205).

Esta representación ambiental en la que los seres humanos aparecen totalmente desligados de la naturaleza, al ser producto del pensamiento moderno y del paradigma cartesiano sobre el que este se cimentó, se ha ido superando gradualmente gracias a los avances obtenidos durante las últimas décadas en campos como la filosofía y la ciencia, los cuales han permitido develar la esencia intrínsecamente compleja de la realidad en cualquiera de sus manifestaciones (MORIN, 1998). Bajo esta renovada forma de representar el mundo, ha cobrado fuerza la visión sistémica del ambiente

[...] en la que se hace evidente que los seres humanos, independientemente del grado de inteligencia y poder científico-técnico que hayan alcanzado, continúan siendo parte de la naturaleza, ya que, entre otras cosas, dependen completamente de sus recursos para sobrevivir. (RICO, 2009, p. 148).

Esta postura integracionista que cada vez se aprecia con mayor frecuencia en el campo ambiental, se puede encontrar en la siguiente intervención:

\footnotetext{
Mujer/24/Farmacia/Nocturna: $\mathrm{Si}$ no creamos conciencia ya mismo la crisis ambiental va empeorar... y el planeta tiene todo el tiempo del mundo para regenerarse, todo el tiempo que quiera, la vida del hombre es la que se va a acabar.
} 
Representaciones sociales sistémicas del ambiente

Ahora bien, de la mano de los trasformaciones del campo ambiental, han surgido los cambios de los habitus lingüísticos de los agentes que allí participan, lo que permite explicar parcialmente desde la sociolingüística porque a pesar del antropocentrismo predominante en sus representaciones, la mayoría de los panelistas entrevistados logra vislumbrar, así sea superficialmente, los vínculos entre los problemas propiamente naturales y las dinámicas socioculturales. Como ya se había señalado, Reigota (1994 apud MOLFI, 2000) se refiere a estas representaciones como globalizantes, pero en este documento se denominaran sistémicas para diferenciarlas del concepto de globalización. Dicha representación se puede apreciar a continuación:

Mujer/25/Química/Nocturna: Es evidente que los seres humanos dependen de la naturaleza para su supervivencia, y que nosotros somos los principales responsables de que la tierra esté así. Por lo tanto, la responsabilidad es de todos, lo que nosotros hagamos acá, afecta a gente al otro lado del mundo, y lo que ellos hagan allá pues nos afecta a nosotros.

Esta visión sistémica del ambiente, es propia de los ambientalistas latinoamericanos, debido a que en el sur del continente las cuestiones ambientales son inseparables de los aspectos económicos, sociales y políticos que afectan la región, y a que la misma sociogénesis de estas organizaciones ha estado estrechamente ligada con otros movimientos sociales como el indígena y el campesino (GÓMEZ, 2001; GUDYNAS, 1992). Dentro de este contexto, el habitus lingüístico de los ambientalistas profesionales, en cuanto sistema de disposiciones socialmente constituido, producto de la trayectoria y de las condiciones sociales en las cuales se ha desarrollado (BOURDIEU, 1985), ha terminado por incorporar tales condiciones objetivas dentro de sus prácticas discursivas, haciendo con ello que conceptos como sistemas complejos e interdisciplinariedad cobren una importancia fundamental, dentro de los criterios que se emplean para evaluar los discursos que circulan dentro del mercado lingüístico propio del campo ambiental suramericano (RICO, 2004).

\section{Representaciones sociales sistémicas del ambiente sencillas y complejas}

El deterioro ambiental en términos generales se puede explicar por causas directas como la deforestación, la minería, las agroindustrias, y la ganadería extensiva, entre muchas otras, y por causas indirectas o subyacentes que son las relacionadas con los problemas estructurales que dan origen a las primeras, como por ejemplo:

a) El antropocentrismo desde el que la naturaleza se concibe solo como un recurso al servicio de la humanidad;

b) El paradigma de desarrollo economicista vigente que promueve la explotación indefinida de los recursos naturales, y que confunde el consumismo con la calidad de vida;

c) Las desiguales relaciones de poder y de intercambio económico entre los estados que poseen la mayor parte de los recursos naturales, que suelen ser pobres, y las potencias económicas que los consumen;

d) La debilidad e ineficacia de las instituciones gubernamentales para expedir y hacer cumplir leyes de carácter ambiental y los altos índices de corrupción que prevalecen en regiones como Latinoamérica.

En ese sentido y con el ánimo de enriquecer la tipología propuesta por el Marcos Reigota, se podría hablar de representaciones sociales del ambiente sistémicas sencillas $y$ complejas. Con las primeras se hace referencia a aquellas representaciones que a pesar de tener en cuenta los elementos socioculturales que hacen parte de las dinámicas ambientales, tienen dificultades para estructurar discursos que den 
cuenta de los intrincados entramados políticos y económicos, que configuran los campos de lucha de las cuestiones ambientales. Con las segundas, se hace alusión a aquellas representaciones que son capaces de describir con claridad, la función que los diferentes componentes de las causas estructurales de los problemas ambientales, juegan en su configuración.

Desde estos referentes, aunque las representaciones sociales sistémicas del ambiente predominan en los discursos examinados, solo la mitad de ellos logra trascender el enfoque sencillo, mientras que la otra mitad se queda a medio camino entre las sencillas y las complejas, tal como lo ilustra la siguiente intervención:

\begin{abstract}
Mujer/19/Quimica/Diurno: Yo pienso que mientras el dinero siga siendo la prioridad en la sociedad, no se van a ver mejoras a nivel ambiental, por el contrario cuestiones como la minería, la gente que ve en el agua un negocio y todo ese tipo de cosas, no van a permitir que las personas realmente se enfoquen en lo que es.
\end{abstract}

Esta representación sistémica del ambiente se encuentra en medio de las dos categorías propuestas, en la medida en que identifica los miopes criterios economicistas que mueven a las sociedades capitalistas, pero sin llegar a dar cuenta de cómo dicha visión del mundo contribuye a configurar escenarios sociopolíticos donde tienen lugar desiguales luchas de poder, que usualmente llevan al deterioro ambiental, en favor de pequeñas minorías.

La diferencia entre las presentaciones sistémicas sencillas y complejas del ambiente se puede apreciar con nitidez, al contrastar la anterior intervención, con la caracterización que se hizo de los discursos del World Rainforest Movement, una organización que trabaja por la defensa de la Amazonía.

De acuerdo con este movimiento, hacen parte las causas subyacentes la pobreza generalizada que se vive en los países miembros de la cuenca y desde luego en la cuenca misma, la cual obliga año tras año a miles de personas a penetrar y destruir la selva con la esperanza de forjarse un mejor futuro; la carencia de una comunidad académica y una estructura científica y tecnológica lo suficientemente fuerte que permita generar los conocimientos necesarios para aprovechar las potencialidades de la selva de forma sostenible; el desmantelamiento gradual que están viviendo los Estados de la región, en el cual son las instituciones gubernamentales ambientales las más afectadas, dando vía libre a las explotaciones ilegales de sus recursos naturales; los modelos de economía de libre mercado inspirados en las teorías neoliberales que desde hace algunos años se vienen aplicando de forma dogmática por los gobernantes de la región; el problema de la deuda externa y los créditos contraídos con instituciones como el Banco Mundial y El Fondo Monetario Internacional, que obliga a los países de la cuenca a explotar sus riquezas naturales, muchas veces de forma insostenible para mantenerse al día con sus acreedores; la intromisión y las presiones ejercidas por las multinacionales sobre los gobiernos de la cuenca para garantizar la explotación de las riquezas del bosque con la mayor rapidez posible, lo que supone hacer las legislaciones ambientales lo más laxas que se pueda y exonerarse de todas las responsabilidades y costos derivados de sus prácticas; etc. (RICO, 2004).

$\mathrm{Al}$ analizar las estrategias discursivas que emplean ambos grupos, se aprecia que mientras los panelistas entrevistados para este trabajo fundamentan la inmensa mayoría de sus argumentos en elementos de carácter racional, apelando muy poco a los recursos emocionales, los ambientalistas profesionales suelen combinar exitosamente el uso de ambos elementos. Dicha fusión, da lugar a estrategias discursivas más efectivas, ya que a algunas personas se las convence más fácilmente apelando a la razón, a otras a los sentimientos y a la mayoría recurriendo simultáneamente a ambos (RICO, 2004). Enseguida se presentan algunos de los pocos fragmentos en los que los estudiantes apelan a las emociones de sus interlocutores: 
Mujer/20/Farmacia/Diurno: La mayoría de las personas están buscando lucrarse económicamente, pero no están ayudando al planeta a sobrevivir, a reconstruir lo que están acabando.

Hombre/18/Química/Diurno: Entonces hasta que no estemos bien mal y no nos veamos ya en una crisis, no vamos a reaccionar.

Mujer/22/Farmacia/Nocturno: Por ejemplo si uno tira un papel a la calle, ya tu hijo te está diciendo "Mami eso no se hace, estas matando el planeta".

\section{Representaciones sociales del ambiente por género, edad y programa de estudio}

Visto desde el enfoque de género, no se apreciaron diferencias significativas entre las representaciones sociales del ambiente de los hombres y mujeres que participaron en los paneles. En cambio sí se observaron algunas discrepancias cuando se analizaron por rangos de edad y programa de estudio. En general, los panelistas de mayor edad le daban más relevancia a los aspectos políticos en la estructuración de sus representaciones sobre el ambiente.

De otra parte, en las representaciones ambientales de los estudiantes de Química Industrial, se evidenció un mayor interés y compromiso que en las de los de Regencia de Farmacia. Esto se explica parcialmente, debido a la dificultad que tienen estos últimos para ver los vínculos entre las problemáticas ambientales (contaminación del aire, de las aguas, de los suelos, perdida de la capa de ozono, etc.) y la salud pública, lo que le quita interés al tópico.

Finalmente, se apreció una fuerte correlación entre el interés y la preocupación que tienen los estudiantes sobre el ambiente, $\mathrm{y}$ las actitudes y comportamientos que asumen frente a las problemáticas ambientales concretas, lo que se traduce en una serie de actividades puntuales como reciclar, ahorrar los recursos naturales, concientizar las personas sobre las cuestiones ambientales, entre muchas otras. Esto permitió corroborar la fuerte relación que existe entre las representaciones sociales y las actitudes y comportamientos de las personas (RODRÍGUEZ, 2002).

\section{Conclusiones}

A lo largo de este trabajo, se apreció como las representaciones sociales, son esenciales para entender el comportamiento de los agentes sociales en cualquier contexto (RODRÍGUEZ, 2002). Ellas permiten acercarse a la comprensión de las formas en que estos perciben el entorno, a las actitudes que asumen bajo la influencia de dichas percepciones y por ende a las estrategias que adoptan para actuar en él (PARALES; VIZCAÍNO-GUTIÉRREZ, 2007).

También se vislumbró como el análisis sociolingüístico del discurso, es una excelente estrategia metodológica (BANCHS, 2000), aunque desde luego no la única, para estudiar las representaciones sociales. Esto debido a que el lenguaje es -junto con las interrelaciones con el contexto donde se mueven los agentes-, causa y consecuencia las representaciones sociales (CIRS, 2010). Dicho en otras palabas, la comunicación es uno de los elementos constituyentes de la realidad social, pero a su vez es un producto de esta misma realidad (BOURDIEU, 1985).

Con respecto a la clasificación de las representaciones sociales del ambiente propuesta por el profesor Marcos Reigota (1994 apud MOLFI, 2000), cabe destacar que existe un elemento común a la gran mayoría de los discursos analizados a lo largo de esta investigación: la posición antropocéntrica que se asume frente a la naturaleza. Esta se hace evidente en casi todos los discursos, cuando se observa que la naturaleza siempre importa como medio para la supervivencia humana, pero no como fin en sí misma.

De igual forma, otra característica común en las representaciones sociales que 
los estudiantes poseen sobre el ambiente, es su visión sistémica del mismo. Esta básicamente consiste en concebir las cuestiones ambientales como producto de la interacción entre las dinámicas socioculturales $\mathrm{y}$ ecosistémicas, en lugar de verlas solamente como cuestiones que poco tienen que ver con el funcionamiento de los grupos humanos, tal como suele ocurrir en las representaciones sociales naturalistas del ambiente (REIGOTA, 1994 apud MOLFI, 2000).

En la caracterización que se hizo de las representaciones sociales sistémicas del ambiente de estos estudiantes, se crearon dos subcategorías. Las representaciones sociales sistémicas sencillas y las complejas. Con las primeras, se hace alusión a aquellas representaciones que alcanzan a identificar los elementos socioculturales que constituyen las cuestiones ambientales, pero que no son capaces de dar cuenta de las complejas interacciones que se estructuran alrededor de los mismos, en los escenarios de lucha de poder que tienen lugar en los campos ambientales. En contraste, con las segundas ocurre todo lo contrario, lo que hace que la calidad de la argumentación alrededor de la influencia que los factores sociales, políticos y económicos tienen sobre las dinámicas ambientales, sea mucho más estructurada. Cabe anotar que la mayoría de las representaciones sociales de los panelistas participantes, cae en la primera de las subcategorías.

Desde los referentes de la sociolingüística (BOURDIEU, 1985), la prevalencia de esta representación sistémica del ambiente, se explica parcialmente en el caso de los estudiantes entrevistados, por la formación integral que muchos de ellos tuvieron en el colegio y por la que están teniendo en la educación superior como parte fundamental de su plan de estudios.

Esta concepción del ambiente constituye una gran ventaja a la hora de emprender acciones concretas para hacerle frente a los diferentes problemas identificados, pues como lo han demostrado innumerables experiencias del pasado, las intervenciones sectoriales en las que solo se le presta atención a una de las dimensiones del problema, resultan muy poco efectivas y sostenibles (GARCÍA, 1994).

De otro lado, en la caracterización sociolingüística que se hizo de estos discursos, se evidenció la predominancia de los argumentos racionales sobre los emocionales. En consecuencia, en la mayor parte de los discursos que se estructuran desde los habitus lingüísticos de estos sujetos, prevalecen las citas de autoridad, las cifras y las referencias a estudios, programas y documentales, así como los conceptos técnicos sobre temas ambientales, apelando muy poco a los recursos retóricos emocionales.

Las generalizaciones que aquí se han hecho, solo son aplicables al contexto sociocultural propio de la CTB, por lo que no se pueden extrapolar a otras instituciones de educación superior. Esto debido a que como ya se ha dicho, las representaciones sociales se construyen contextualmente en espacios y tiempos específicos (RODRÍGUEZ, 2007). 


\section{Referencias}

ÁNGEL, Augusto. Medio ambiente e interdisciplina: utopías y realidades. In: SEMINARIO INTERNACIONAL UNIVERSIDAD Y MEDIO AMBIENTE EN AMÉRICA LATINA Y EL CARIBE, 2., 2001, Cali. Memorias... Cali: [s. n.], 2001. p. 81.

ALFONSO, Ibette. La teoría de las representaciones sociales. Valencia: Clínica Psicológica V. Mars - Sueca, 2011. Disponible en: <http://www.psicologia-nline.com/articulos/2007/representaciones_sociales.shtml.> Acceso en: 07 nov. 2014.

BANCHS, María. Aproximaciones procesuales y estructurales al estudio de las representaciones sociales. Pepers on Social Representations, London, v. 9, p. 3.1-3.15, 2000.

BERMÚDEZ, Olga María. Cultura y ambiente: la educación ambiental contexto y perspectivas. Bogotá: IDEA-UN, 2003.

BOURDIEU, Pierre. Cuestiones de sociología. Madrid: Istmo, 2000.

BOURDIEU, Pierre. ¿Qué significa hablar? Madrid: Akal, 1985.

BOURDIEU, Pierre; WACQUANT, Loic. Respuestas por una antropología reflexiva. México: Grijalbo, 1995.

CARRISOZA, Julio. ¿Qué es ambientalismo? La visión ambiental compleja. Bogotá: PNUMA-IDEA-CEREC, 2000.

CHRISTIDOU, Vasilla; DIMOPOULOS, Kostas; KOULAIDIS, Vasilis. Constructing social representations of science and technology: the role of metaphors in the press and the popular scientific magazines. Public Understanding of Science, London, v. 13, p. 347-362, 2004.

CISRS - CONFERENCIA INTERNACIONAL SOBRE REPRESENTACIONES SOCIALES: REPRESENTACIONES, TRASMISIÓN DE SABERES Y TRANSFORMACIONES SOCIALES, 10., 2010, Túnez. Disponible en: <http://www.construccionismosocial.net/2009/09/10aconferencia-internacional-sobre.html>. Acceso en: 14 jul. 2013.

CTB - Corporación Tecnológica de Bogotá. Misión y visón. Bogotá: CTB, 2013. Disponible en: <http://portal.ctb.edu.co/index. php/mision-y-vision>. Acceso en: 23 mar. 2013.

FERREIRA, Rosana Louro. Representaciones sociales de medio ambiente y educación ambiental de docentes universitarios(as). Tópicos en Educación Ambiental, México, DF., v. 4, n. 10, p. 22-36, 2002.

GARCÍA, Rolando. Interdisciplinariedad y sistemas complejos. In: LEFF, Enrique (Ed.). Ciencias sociales y formación ambiental. Barcelona: Gedisa, 1994. p. 85-112.

GÓMEZ, Luis Jair. Movimiento e institucionalidad política ambiental internacional: génesis y bases conceptuales. Gestión y Ambiente, Medellín, v. 4, n. 1, p. 59-69, jul. 2001.

GUDYNAS, Eduardo. Una extraña pareja: Ios ambientalistas y el Estado en América Latina. Ecología Política, Barcelona, n. 3, p. 51-64, 1992.

MOLFI, Eneida María. Deconstrucción de las representaciones sobre el medio ambiente y la educación ambiental. Tópicos en Educación Ambiental, México, DF., v. 2, n. 4, p. 33-40, 2000.

MORIN, Edgar. Introducción al pensamiento complejo. Barcelona: Gedisa, 1998.

PARALES, Carlos José; VIZCAínO-GUTIÉRREZ, Milciades. Las relaciones entre actitudes y representaciones sociales: elementos para una integración conceptual. Revista Latinoamericana de Psicología, Bogotá, v. 39, n. 2, p. 351-361, 2007.

PERERA, Maricela de la Caridad. A propósito de las representaciones sociales: apuntes teóricos, trayectoria y actualidad. 1999. Disponible en: http://bibliotecavirtual.clacso.org.ar/ar/libros/cuba/cips/caudales05/Caudales/ARTICULOS/ArticulosPDF/02P075. pdf >. Acesso en: 06 nov. 2014.

PERERA, Maricela de la Caridad. Sistematización crítica de la teoría de las representaciones sociales. 2005. Tesis (Doctorado en Ciencias Psicológicas), Centro de Investigaciones Psicológicas y Sociológicas, Ministerio de Ciencia, Tecnología y Medio Ambiente. La Habana, 2005. 
REIGOTA, Marcos. Herramientas metodológicas para el trabajo en educación ambiental. 2001. Disponible en: <http://www. virtual.unal.edu.co/cursos/DEA/2007225/lecciones/capitulo2/04-herramientasmetodologicas4.htm>. Acceso en: 07 jul. 2009.

RICO, Alejandro. Ambientalismo y religión: vínculos entre la crisis ambiental contemporánea y el legado judeocristiano. Gestión y Ambiente, Medellín, v. 12, n. 3, p 143-154, ago./dic. 2009.

RICO, Alejandro. Análisis sociolingüístico de los discursos ambientales sobre la Amazonía. Revista Gestión y Ambiente, Medellín, v. 7, n. 2, p. 5-14, dic. 2004.

RODRÍGUEZ, Tanía. Representar para actuar, representar para pensar. Breves notas metodológicas. In: PALACIO, Celia. Cultura, comunicación y política. Guadalajara, CUCSH-UdeG, 2002. p. 25-41.

RODRÍGUEZ, Tanía. Sobre el carácter contextual de las representaciones sociales. In: JORNADA INTERNACIONAL, 5., 2007 e CONFERÊNCIA BRASILEIRA SOBRE REPRESENTAÇÕES SOCIAIS, 3., 2007, Brasilia. Anais... Brasília, DF: [s. n.], 2007. p. 1-11.

WHITE, Lynn. The historical roots of our ecological crisis. Science, n. 155, p. 1203-1207, 1967.

Recibido en: 14.12.2014

Aprobado en: 10.03.2015

Alejandro Rico Méndez es sociólogo de la Universidad Nacional de Colombia, especialista en Docencia Universitaria y Maestro en Educación. 